POS PROCEEDINGS

\title{
CUORICINO status and CUORE prospects
}

\section{Paolo Gorla*t}

Lab. Naz. Gran Sasso INFN, Italy

E-mail: paolo.gorla@lngs.infn.it

R. Ardito ${ }^{1,2}$, C. Arnaboldi ${ }^{1}$, D. R. Artusa ${ }^{3}$, F. T. Avignone $\mathrm{III}^{3}$, M. Balata ${ }^{4}$, I. Bandac ${ }^{3}$,

M. Barucci ${ }^{5}$, J.W. Beeman ${ }^{6}$, F. Bellini ${ }^{16}$, C. Brofferio ${ }^{1}$, C. Bucci ${ }^{4}$, S. Capelli ${ }^{1}$,

L. Carbone ${ }^{1}$, S. Cebrian ${ }^{7}$, M. Clemenza ${ }^{1}$, C. Cosmelli ${ }^{16}$, O. Cremonesi ${ }^{1}$,

R. J. Creswick ${ }^{3}$, I. Dafinei ${ }^{16}$, A. de Waard ${ }^{8}$, M. Diemoz ${ }^{16}$, M. Dolinski ${ }^{6,11}$,

H. A. Farach ${ }^{3}$, F. Ferroni ${ }^{16}$, E. Fiorini ${ }^{1}$, S.J. Freedman ${ }^{6,11}$, C. Gargiulo ${ }^{16}$,

E. Guardincerri ${ }^{10}$, A. Giuliani ${ }^{9}$, T.D. Gutierrez ${ }^{6}$, E. E. Haller ${ }^{6,13}$, K. Heeger ${ }^{14}$,

I. G. Irastorza ${ }^{7}$, E. Longo ${ }^{16}$, G. Maier ${ }^{2}$, R. Maruyama ${ }^{11}$, S. Morganti ${ }^{16}$, S. Nisi ${ }^{4}$,

C. Nones ${ }^{1}$, E. B. Norman ${ }^{15}$, A. Nucciotti ${ }^{1}$, E. Olivieri ${ }^{5}$, P. Ottonello ${ }^{10}$, M. Pallavicini ${ }^{10}$, V. Palmieri ${ }^{14}$, M. Pavan ${ }^{1}$, M. Pedretti ${ }^{9}$, G. Pessina ${ }^{1}$, S. Pirro ${ }^{1}$, E. Previtali ${ }^{1}$,

L. Risegari ${ }^{5}$, C. Rosenfeld ${ }^{3}$, S. Sangiorgio ${ }^{9}$, M. Sisti ${ }^{1}$, A. R. Smith ${ }^{6}$, L. Torres $^{1}$,

G. Ventura ${ }^{5}$, N. $X_{u^{6}}$, and L. Zanotti ${ }^{1}$ 
${ }^{1}$ Dipartimento di Fisica dell'Università di Milano-Bicocca e Sezione di Milano dell'INFN, Milano I-20126, Italy

${ }^{2}$ Dipartimento di Ingegneria Strutturale del Politecnico di Milano, Milano I-20133, Italy

${ }^{3}$ Dept.of Physics and Astronomy, University of South Carolina, Columbia, South Carolina, USA 29208

${ }^{4}$ Laboratori Nazionali del Gran Sasso, I-67010, Assergi (L'Aquila), Italy

${ }^{5}$ Dipartimento di Fisica dell'Università di Firenze e Sezione di Firenze dell'INFN, Firenze I-50125, Italy

${ }^{6}$ Nuclear Science Division, Lawrence Berkeley National Laboratory, Berkeley, CA 94720, USA

${ }^{7}$ Laboratorio de Fisica Nuclear y Altas Energias, Universidàd de Zaragoza, 50009 Zaragoza, Spain

${ }^{8}$ Kamerling Onnes Laboratory, Leiden University, 2300 RAQ, Leiden, The Netherlands

${ }^{9}$ Dipartimento di Fisica e Matematica dell'Università dell'Insubria e Sezione di Milano dell'INFN, Como I-22100, Italy

${ }^{10}$ Dipartimento di Fisica dell'Università di Genova e Sezione di Genova dell'INFN, Genova I-16146, Italy

${ }^{11}$ Department of Physics, University of California, Berkeley, CA 94720, USA

${ }^{12}$ Department of Materials Science and Mineral Engineering, University of California, Berkeley, CA 94720, USA

${ }^{13}$ Physics Division, Lawrence Berkeley National Laboratory, Berkeley, CA 94720, USA

${ }^{14}$ Laboratori Nazionali di Legnaro, I-35020 Legnaro ( Padova ),Italy

${ }^{15}$ Lawrence Livermore National Laboratory, Livermore, California, 94550, USA

${ }^{16}$ Dipartimento di Fisica dell'Università di Roma e Sezione di Roma 1 dell'INFN, Roma I-16146, Italy

CUORE is a 1-ton experiment to search for Neutrinoless Double Beta Decay of $\mathrm{Te}^{130}$ using 988 $\mathrm{TeO}_{2}$ bolometers. It aims at reaching a sensitivity on the effective neutrino mass of the order of few tens of meV. CUORICINO, a single CUORE tower running since 2003, plays an important role as a standing alone experiment and for developing the future CUORE setup. Present results already achieved and studies that are underway are here presented and discussed. 


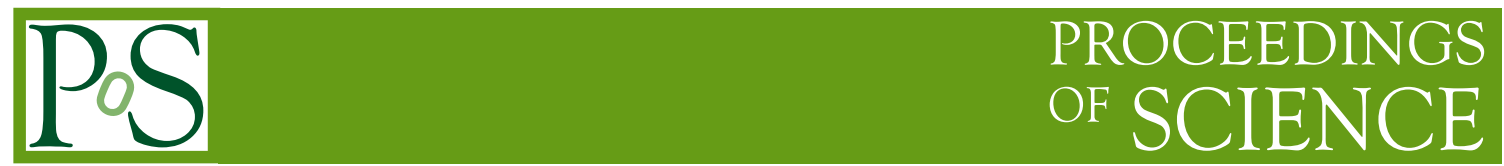

International Europhysics Conference on High Energy Physics July 21st - 27th 2005

Lisboa, Portugal

*Speaker.

${ }^{\dagger}$ A footnote may follow. 


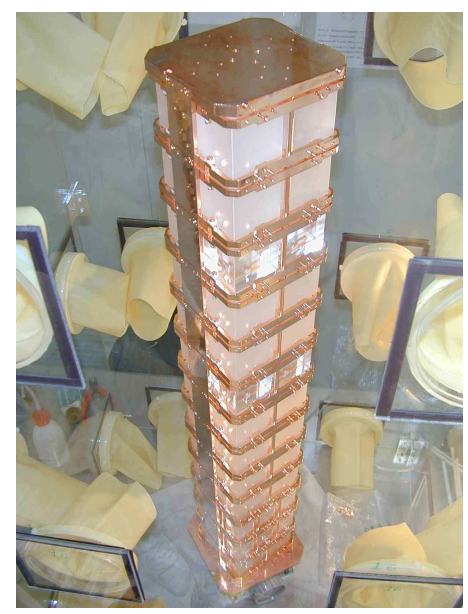

Figure 1: The CUORICINO tower inside a nitrogen-fluxed glove-box, during the final assembly of the detector.

\section{Introduction}

The evidence of a neutrino rest mass represents one of the most exciting discovery in the field of particle physics. The discovery of the neutrinoless Double Beta Decay (0v-DBD), however, will provide not only the ultimate answer about the nature (Dirac or Majorana) of the neutrino, but will also allow a sensitivity on the mass down to few meV. The use of the bolometric technique offers the unique possibility to investigate different $0 v$-DBD candidates with a considerable high energy resolution, needed for future experiments. The CUORE experiment [1], to search $0 v$-DBD of ${ }^{130} \mathrm{Te}$, will start its assembling phase in 2006 and it aims to reach a sensitivity on Majorana mass better than $50 \mathrm{meV}$. CUORICINO represent not only the first stage of CUORE, but also the most massive $0 v$-DBD Experiment presently running.

\section{Cuoricino setup}

CUORICINO [2] is an array of 62 crystals of $\mathrm{TeO}_{2}$ with a total active mass of $40.7 \mathrm{~kg}$, that corresponds to a mass of ${ }^{130} \mathrm{Te}$ of $\sim 11 \mathrm{~kg}$. The tower is located inside the cryostat situated in the Hall A of Laboratori Nazionali del Gran Sasso (LNGS) of INFN. CUORICINO's 62 crystals are arranged in a tower made by 13 planes (Fig. 1), 11 of them are filled with 4 cubes of $5 \mathrm{~cm}$ side while the other two with 9 crystals $3 \times 3 \times 6 \mathrm{~cm}^{3}$ each. Four $3 \times 3 \times 6 \mathrm{~cm}^{3}$ crystals are enriched, two of which in ${ }^{128} \mathrm{Te}, 82.3 \%$ isotopic abundance, and the other two in ${ }^{130} \mathrm{Te}$, isotopic abundance of 75 $\%$.

All the materials composing the detector were selected to be low contaminated with radioactive isotopes. To avoid external vibrations to reach the detectors the tower is mechanically decoupled from the cryostat through a steel spring. In order to shield against the radioactive contaminants from the materials of the refrigerator, a $1.2 \mathrm{~cm}$ shield of Roman lead with ${ }^{210} \mathrm{~Pb}$ activity of $<4 \mathrm{mBq} / \mathrm{kg}$ [3] is framed around the array to reduce the activity of the thermal shields. The cryostat is externally shielded by means of two layers of lead of $10 \mathrm{~cm}$ minimal thickness each. The background due to 


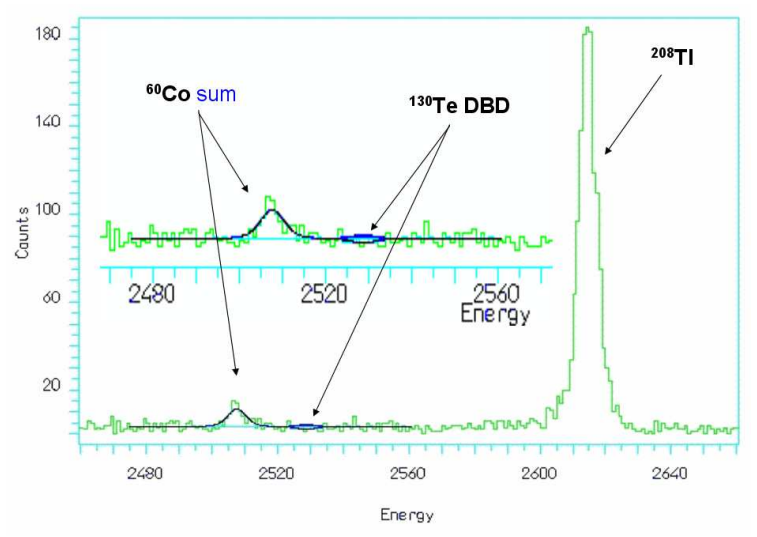

Figure 2: CUORICINO spectrum in the $0 v$ DBD energy region.

environmental neutrons is reduced by a layer of Borated Polyethylene of $10 \mathrm{~cm}$ minimum thickness. The refrigerator operates inside a Plexiglass anti-radon box flushed with clean $\mathrm{N}_{2}$ and inside a Faraday cage to reduce electromagnetic interferences. Thermal pulses are recorded by neutron transmutation doped Ge thermistors thermally coupled to each crystal. CUORICINO is operated at a temperature of $\sim 8 \mathrm{mK}$ with a spread of $\sim 1 \mathrm{mK}$. The energy calibration is performed before and after each subset of runs, which lasts about a month, by exposing the array to two thoriated tungsten wires inserted in immediate vicinity of the refrigerator.

\section{Physics results}

CUORICINO first measurement started in March 2003 and ended in of October 2003. After a substantial operation of maintenance in April 2004 the second run of CUORICINO started. The average pulse Amplitude is of $215 \mu \mathrm{V} / \mathrm{MeV}$ for the $5 \times 5 \times 5$ crystals and $430 \mu \mathrm{V} / \mathrm{MeV}$ for the $3 \times 3 \times 6 \mathrm{~cm}^{3}$ crystals. The average resolution FWHM is $7.5 \pm 2.9 \mathrm{keV}$ for the bigger size and of $9.6 \pm 3.5 \mathrm{keV}$ for the small size crystals. The duty cycle of the experiment, since August 2004 is $\sim 73 \%$. Discarding the time needed for energy calibration measurement (3 days every 3-4 weeks) the total background live time is $63 \%$. The total background spectra collected up to July 2005, corresponding to a total statistic of $5.87 \mathrm{~kg}\left(\right.$ of $\left.{ }^{130} \mathrm{Te}\right)$.year, is presented in Fig. 2. Apart the ${ }^{60} \mathrm{Co}$ sum line and the ${ }^{208} \mathrm{Tl}$ line, no other unexpected peak if found near the $2528 \mathrm{keV} 0 \mathrm{vDBD}$ region of ${ }^{130} \mathrm{Te}$. The obtained lower limit on the $0 v \mathrm{DBD}$ of ${ }^{130} \mathrm{Te}$ is of $2.0 \times 10^{24} \mathrm{y}(90 \%$ C.L.) This limit leads to a constraint on the electron neutrino effective Majorana mass ranging from 0.2 to $1.0 \mathrm{eV}$, depending on the nuclear matrix elements considered in the computation.

\section{The CUORE experiment}

The CUORE detector will consist of an array of $988 \mathrm{TeO}_{2}$ bolometers arranged in a cylindrical configuration of 19 towers containing 52 crystals each (Fig. 3), for a total mass of $\sim 741 \mathrm{~kg}$. Each of these towers is a CUORICINO-like detector consisting of 13 modules, 4 detectors each. Assuming a background of $\mathrm{B}=0.01 \mathrm{c} / \mathrm{keV} / \mathrm{kg} / \mathrm{y}$, achievable with a slight improvement of the current available 


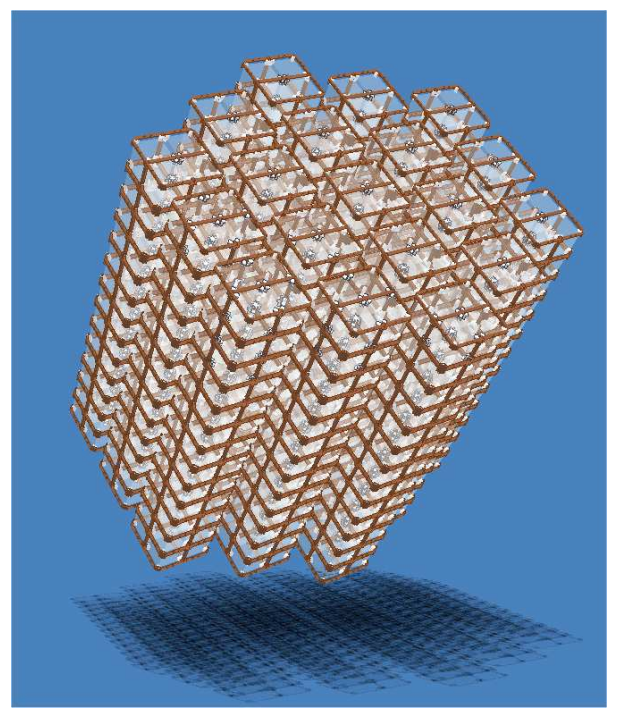

Figure 3: The CUORE detector (cylindrical-shaped) built of 19 CUORICINO-like tower.

material selection and cleaning techniques, and an energy resolution $\Gamma(2.5 \mathrm{MeV})=5 \mathrm{keV}$, we get a sensitivity $\mathrm{S}_{0 v}$ on the half life $\left(90 \%\right.$ C.L.) of $5.8 \cdot 10^{25} \sqrt{t}$ years $\left(4.1 \cdot 10^{25} \sqrt{t}\right.$ years for $\Gamma=10$ $\mathrm{keV})$, which in 5 years of statistics would provide $\left|\left\langle m_{v}\right\rangle\right|$ bounds in the range $0.024-0.13 \mathrm{eV}$. However, the R\&D to be carried out in CUORE, if successful, would provide a value of $\mathrm{B} \sim 0.001$ $\mathrm{c} / \mathrm{keV} / \mathrm{kg} / \mathrm{y}$, i.e. a detection sensitivity of $\mathrm{S}_{0 v} \sim 1.86 \cdot 10^{26} \sqrt{t}$ years $\left(1.2 \cdot 10^{26} \sqrt{t}\right.$ years for $\Gamma=10$ $\mathrm{keV}$ ), or $\left|\left\langle m_{v}\right\rangle\right|$ bounds in the range $\sim 0.016-0.085 \mathrm{eV}$ in 5 years. $\mathrm{TeO}_{2}$ crystals made with ${ }^{130} \mathrm{Te}$ enriched material have been already operated in MiDBD and CUORICINO, making an enriched CUORE a feasible option. Assuming a $95 \%$ enrichment in ${ }^{130} \mathrm{Te}$ and a background level of $\mathrm{b}=0.001$ $\mathrm{c} / \mathrm{keV} / \mathrm{kg} / \mathrm{y}$, the sensitivity would become $\mathrm{S}_{0 v} \sim 8.32 \cdot 10^{26} \sqrt{t}$ years. For an exposure of 5 years, the corresponding $\left|\left\langle m_{v}\right\rangle\right|$ bounds would range from $8 \mathrm{meV}$ to $45 \mathrm{meV}$ depending on the nuclear matrix element calculations.

\section{References}

[1] C. Arnaboldi et al., Nucl. Instrum. Meth. A 518 (2004) 775

[2] C. Arnaboldi et al., Phys. Rev. Lett. 95, 142501 (2005) [arXiv:hep-ex/0501034].

[3] A. Alessandrello et al., Nucl. Instr. and Meth. B 142(1998)163 